\title{
Incentivizing research into the effectiveness of medical devices
}

\author{
Michael Drummond $^{1,2} \cdot$ Rosanna Tarricone $^{2} \cdot$ Aleksandra Torbica $^{2}$
}

Published online: 4 August 2016

(C) Springer-Verlag Berlin Heidelberg 2016

\section{Introduction}

Medical devices (MDs) often obtain market authorization with much less clinical evidence than other health technologies, especially pharmaceuticals. This is due to a number of reasons. First, in contrast to pharmaceuticals, there is no legal requirement to conduct adequately controlled clinical studies, other than for 'high-risk' devices in some jurisdictions. In the US for example, high-risk devices and innovative lower-risk devices are required to demonstrate 'reasonable assurance of safety and effectiveness', which may imply clinical evidence based on randomized studies in many instances. In contrast, in the EU the requirement is to demonstrate adequate performance and safety, which can often be achieved by conducting observational studies such as registries [1,2].

Secondly, the devices industry comprises many small and medium-size enterprises (SMEs), which would find the cost of conducting clinical studies, especially randomized controlled trials, prohibitive. However, although some larger manufacturers do undertake clinical studies of some of their products, manufacturers with similar products (called 'fast-followers') can often claim 'substantial equivalence' to a product that already has market authorization, thus avoiding the need to conduct costly and timeconsuming clinical studies. Since regulatory agencies often accept these claims of equivalence, for example under the

Michael Drummond

mike.drummond@york.ac.uk

1 Centre for Health Economics, University of York, Alcuin A Block, Heslington, York YO10 5DD, UK

2 Centre for Research on Health and Social Care Management (Cergas), Università Bocconi, Milan, Italy 510(k) process in the US [3], this further reduces the incentives for manufacturers to conduct expensive clinical studies. Therefore, although device manufacturers have patent protection, they are often not granted data exclusivity in the same way as pharmaceutical manufacturers.

Finally, unlike pharmaceuticals, devices are often modified once on the market, meaning that even if clinical evidence was available for the original version of the product, it may not necessarily be available for the version currently being marketed. For example in the US, one analysis showed that for 77 original market authorization applications for cardiac implantable electronic devices (e.g., pacemakers, implantable cardioverter-defibrillators) since 1979, the FDA approved 5829 'supplements' reflecting product modifications in the period up until 2012. Of course, many of these product modifications were minor and unlikely to affect the performance of the device, but $37 \%$ involved a change to the device's design. In the vast majority of these cases the FDA deemed that new clinical data were not necessary for approval [4].

The lack of clinical evidence prior to product launch, especially evidence of comparative effectiveness, limits the possibilities for health technology assessment [2]. However, it should be remembered that clinical evidence can be gathered both pre-market (i.e., through conducting controlled clinical trials in an experimental setting), and postmarket, through clinical studies undertaken in regular clinical practice. Post-market effectiveness research may be more important for MDs than pharmaceuticals, as the performance of the device often depends on the interaction with the user (the so-called learning curve) [5].

This suggests that solutions to the problem of inadequate clinical evidence should address the issue of conducting clinical research in both the pre- and post-market phase. In this editorial we consider ways in which MD manufacturers 
could be incentivized to produce more clinical evidence to facilitate health technology assessments, including economic evaluations.

\section{Changing the requirements for clinical studies in the pre-marketing phase}

One option would be to strengthen the requirements for conducting clinical studies in the pre-marketing phase. However, this can have disadvantages. Shuren and Califf [6] note that the greater evidentiary burden imposed by the Food and Drug Administration may create disincentives for manufacturers to bring important medical devices to the United States, or may delay access to devices. For example, they point out that the first transcatheter aortic valve replacement device was available for clinical use in Europe several years before it was available in the United States. However, they also point out that there are examples of unsafe and ineffective devices marketed in Europe that never made it to the US market [7].

In most jurisdictions, the requirements for clinical studies in the pre-marketing stage depend on the level of risk associated with the device, although the precise requirements vary across jurisdictions. It is appropriate that the emphasis is on the clinical assessment of 'high risk' devices, but the manufacturers of some devices classified as 'moderate risk' (Class II in the US), such as hip and knee prostheses, are not necessarily required to conduct clinical studies. Kesselheim and Rajan [8] point out that the FDA cleared a metal-on-metal hip under the $510(\mathrm{k})$ pathway that was later shown to posses a high risk of wear, corrosion and metal release into the bloodstream.

Therefore, there may be a case for strengthening the requirements for clinical studies in the pre-marketing phase, based on safety considerations, which in turn would improve the evidence base for health technology assessment (HTA). This could involve requirements for randomized controlled trials (RCTs) or high quality observational studies, depending on the category of device. In some cases a traditional RCT, randomizing patients to treatment or control, may be difficult or impossible to conduct. However, it may be possible to delay treatment for some patients, or to take advantage of different rates of diffusion of particular devices in different geographical areas. The most critical issue is that of determining the evidentiary needs at each stage of the regulatory and reimbursement process. In Canada, an innovative program called EXCITE, seeks to coordinate the process of evidence development for MDs in both the pre- and postmarket phases [9]. Under this program device manufacturers can ask for advice on the studies required to expedite the market approval and reimbursement of their product. A similar initiative, known as Shaping European Early Dialogues (SEEDS), has been recently launched in Europe through an international project financed by the European Commission (www.earlydialogues.eu, accessed on 18 July 2016). The aim of the SEED project was to conduct pilots on early dialogues between HTA agencies and developers of health products (pharmaceuticals and medical devices) whose products were at the development stage. A total of 11 pilot early dialogues have been completed ( 8 on drugs and 3 on medical devices). The SEED consortium is expected to release its recommendations for a permanent model for conducting early dialogues in Europe in late 2016.

In 2012, the European Commission adopted a package of measures on innovation in health. The package consisted of a communication and two regulation proposals to revise existing legislation. Specifically, it contained proposals to improve the traceability of medical devices throughout the supply chain and updated risk classification rules, dividing medical devices into four different risk categories. The proposed revisions affected all kinds of medical devices including in vitro diagnostic medical devices, from homeuse items like sticking plasters, pregnancy tests and contact lenses, to X-ray machines, pacemakers, breast implants, hip replacements and HIV blood tests.

The aim of the revisions was to ensure a consistently high level of health and safety protection for EU citizens using these products, the free and fair trade of the products throughout the EU and to ensure that EU legislation is adapted to the significant technological and scientific progress in this sector over the last 20 years. Revisions included extending the scope for legislation, better supervision of independent assessment bodies, clear rights for manufacturers/distributors and stronger requirements for medical evidence aimed at strengthening clinical evidence, not necessarily experimental trials, in the pre-market phase and in the post-marketing phase through a "post market clinical follow up plan and a medical device vigilance system" (http://ec.europa.eu/growth/sectors/ medical-devices/regulatory-framework/revision_en; accessed on 23 July 2016).

The other main consideration in the pre-marketing phase concerns the level of evidence required for devices manufactured by 'fast-followers'. Independently of any concerns about efficacy and safety, it might be argued that, in order to incentivize manufacturers to conduct clinical studies, manufacturers should be granted some measure of data exclusivity, in order to reward them for investing in evidence generation. Sorenson and Drummond [10] argued that fast-followers could be required to generate the same clinical evidence as for other devices already on the market, unless there is compelling evidence of their comparable manufacture. However, this could result in a waste of resources in conducting clinical studies that are not 
necessary, raising clinical development costs and denying the health care system the benefits that arise from the price competition that fast-follows generate. In addition, the larger companies with the resources to undertake higher quality clinical studies might use this requirement as a barrier to entry for smaller companies.

\section{Clarifying the criteria for 'substantial equivalence'}

Given the issues identified above, it is likely that some role for claiming 'substantial equivalence' will remain. However, the grounds for claiming 'substantial equivalence' need to be carefully defined. Evidence from the $510(\mathrm{k})$ program in the US suggests that some products granted equivalence were found to be not truly equivalent in the long run [8]. This suggests that under programs such as $510(\mathrm{k})$, there should also be a provision for collecting follow-up clinical data in order to verify that the decision to grant substantial equivalence was correct. The same approach could also be followed in verifying whether product modifications lead to changes in the performance of MDs [4].

\section{Strengthening the arrangements for post-market research}

Many of the comments above suggest that the most promising approach might be to strengthen the arrangements for post-market research. Not only would this avoid the costs and delays in patient access resulting from more stringent requirements for pre-market research, it would produce more evidence on the effectiveness of MDs in regular clinical practice. Many clinical registries for MDs already exist, but many are of inadequate quality, or are not useful for health technology assessment. One important, and generally agreed, improvement is the use of a unique device identifier, which is being phased in by the FDA and is also proposed in the EU. This will greatly facilitate the traceability of MDs and help monitor safety and effectiveness [11]. The recent European Union MedtecHTA Project made further recommendations concerning the design of registries to facilitate the assessment of comparative effectiveness. Ideally, registries should include more than one device, routinely collect information on possible confounding factors in order to facilitate the analysis of observational data, collect data on treatment patterns and resource use over time in order to monitor learning effects and stratify patients by level of risk [12].

In order to bring about these changes, jurisdictions need to develop a coordinated approach to the initiation, design and analysis of post-market research for MDs. Such initiatives have been undertaken in a number of jurisdictions, known variously as 'coverage with evidence development' in the US, 'field evaluations' in Canada, or 'approval with research' in the United Kingdom. Typically, these schemes are initiated by the main payer for health care in the jurisdiction concerned, such as the government or social insurance system, although the arrangements for funding differ from jurisdiction to jurisdiction. The advantages and disadvantages of these initiatives, known collectively as 'performance-based risk-sharing agreements' have been reviewed by Garrison et al. [13] and Drummond [14]. Rothery et al. [15] discuss a number of the methodological issues in the design of these programs and how the nature of the evidence collected and the nature of the requirement imposed on manufacturers may change as the evidence base on the device becomes more mature.

Post-market schemes also present the opportunity to address some of the issues surrounding the incentives to conduct research, which are central to this paper. Rather than relying mainly on a single manufacturer, typically the first to market, to bear all the costs of undertaking the research and then granting others substantial equivalence at minimal cost, these schemes could facilitate cost-sharing for research. If, on approval to market, each device was entered into the scheme after the conditions of substantial equivalence were met, the costs of further research could be shared among manufacturers, either in relation to the number of devices marketed, or in relation to their market share by value. Therefore, although the costs of research may still fall disproportionately on some manufacturers, this will be partly offset by the revenue that they earn.

In addition, by entering each new device into a postmarket scheme when it is granted market authorization, opportunities to gather evidence on the superior effectiveness of new devices compared with current standard of care, plus comparative effectiveness and safety among similar devices, are maximized and prior decisions to allow market entry of new devices based on the belief of substantial equivalence can be verified.

There is no simple solution to rectifying the clinical evidence gap for medical devices. In particular, it would be too simplistic to treat them in the same way as pharmaceuticals. However, we believe that the proposals made in this paper offer a way forward.

\section{References}

1. Kramer, D.B., Xu, S., Kesselheim, A.S.: Regulation of medical devices in the United States and European Union. N. Engl. J. Med. 366, 848-855 (2012)

2. Tarricone, R., Ferrè, F., Torbica, A., Drummond, M.F.: Generating appropriate clinical data for value assessment of medical 
devices: what role does regulation play? Expert Rev. Pharmacoecon. Outcomes Res. 14(5), 707-718 (2014)

3. Fronsdal, K., Pichler, F., Mardhani-Bayne, L., HenshallC, Rottingen J.A., Morland, B., et al.: Interaction initiatives between regulatory, health technology assessment and coverage bodies, and industry. Int. J. Technol. Assess. Health Care 28, 374-381 (2012)

4. Rome, B.N., Kramer, D.B., Kesselheim, A.S.: FDA approval of cardiac implantable electronic devices via original and supplement premarket approval pathways. J. Am. Med. Assoc. 311, 385-391 (2014)

5. Drummond, M.F., Griffin, A., Tarricone, R.: Economic evaluation for devices and drugs. Same or different? Value in Health 12(4), 402-404 (2009)

6. Shuren J, Califf RM: Need for a national evaluation system for health technology. JAMA (2016). doi:10.1001/jama.2016.8708

7. US Food and Drug Administration: Unsafe and ineffective devices approved in the EU that were not approved in the US (2016). http://www.elsevierbi.com/ /media/Supporting\% 20Documents/The\%20Gray\%20Sheet/38/20/FDA_EU_Devices_ Report.pdf. Accessed 18 July 2016

8. Kesselheim, A.S., Rajan, P.V.: Regulating incremental innovation in medical devices. Br. Med. J. 349, g5303 (2014). doi:10. 1136/bmj.g5303
9. Tsoi, B., O’Reilly, D., Masucci, L., Drummond, M.F., Goeree, R.: Harmonization of HTA-based reimbursement and regulatory approval activities: a qualitative study. J. Popul. Ther. Clin. Pharmacol. 22(1), e78-e89 (2015)

10. Sorenson, C., Drummond, M.F.: Improving medical device regulation: the US and Europe in perspective. Milbank Q. 92(1), 114-150 (2014). doi:10.1111/1468-0009.12043

11. Food and Drug Administration: Rockville, MD (2016). http:// www.fda.gov/MedicalDevices/DeviceRegulationandGuidance/ UniqueDeviceIdentification/. Accessed 28 June 2016

12. Tarricone R, Torbica A, Drummond, MF for the MedtecHTA Project Group: Key recommendations from the MedtecHTA project (2016). www.medtechta.eu. Accessed 19 July 2016

13. Garrison, L.P., Towse, A., Briggs, A., de Pouvourville, G., Greuger, J., Mohr, P.E., Severens, J.L., Siviero, P., Sleeper, M.: Performancebased risk-sharing arrangements-good practices for design, implementation and evaluation. Value Health 16, 703-719 (2013)

14. Drummond, M.F.: When do performance-based risk-sharing arrangements make sense? Eur. J. Health Econ. 16(6), 569-571 (2015). doi:10.1007/s10198-015-0683-Z

15. Rothery C, Epstein D, Claxton K, Palmer S, Taylor R, Tarricone $\mathrm{R}$, Sculpher M: Characterising uncertainty in the assessment of medical devices and determining future research needs (2016). www.medtechta.eu. Accessed 19 July 2016 